\section{Tinnitus: Retraining bei deutlicher Beeinträchtigung besonders wirksam}

\section{Bei chronischem Tinnitus steht der individuelle Umgang mit den Ohrgeräuschen im Vordergrund der Therapie. Die Langzeitwirksamkeit eines tagesklinischen intensiven Retrainings wurde jetzt an der Charité in Berlin untersucht.}

F ir eine Studie zur Wirksamkeit der Tinnitus-Retraining-Therapie (TRT) wurden 237 Patienten mit einem mindestens drei Monate anhaltenden Tinnitus rekrutiert, viele von ihnen litten aber schon über zwei Jahre an den Ohrgeräuschen, 151 davon bilateral. Die multidisziplinäre TRT dauerte sieben Tage und bestand aus Elementen wie dem Erlernen der Progressiven Muskelrelaxation nach Jacobsen, Akustischer Therapie, Physiotherapie, Schulung, Trainieren der Lenkung der selektiven Aufmerksamkeit sowie Veränderung der Einschätzung, der mentalen Haltung und dem Verhalten gegenüber dem eigenen Tinnitus. Um die Effektivität dieses Ansatzes zu überprüfen, wurden Stressvariablen und die Tinnitusspezifische Belastung vor der Therapie und direkt danach oder nach drei, sechs und zwölf Monaten untersucht. 192 der Patienten erhielten die TRT, die übrigen waren auf einer Warteliste und dienten als Kontrollgruppe für die Drei-MonatsErgebnisse. Für den direkten Vergleich zu diesem Zeitpunkt wurden 45 Patienten der Therapie- und 45 Patienten der Kontrollgruppe herangezogen.

In der Kontrollgruppe änderten sich der Schweregrad des Tinnitus nach dem Tinnitus-Questionnaire (TQ), die Werte des Perceived Stress-Questionnaire (PSQ) sowie der Allgemeinen Depressionsskala (ADS) über drei Monate nicht, während in der TRT-Gruppe sowohl die Beeinträchtigung nach dem TQ als auch nach der ADS abnahmen. Für beide Parameter war der Unterschied zwischen den beiden Gruppen nach drei Monaten signifikant. Im Langzeitverlauf bis zu zwölf Monaten zeigte sich ein anhaltender mäßiggradiger Effekt der TRT auf die Ergebnisse des TQ der 192 Patienten der Therapiegruppe. Der PSQ-Wert zeigte einen Trend zur Besserung über ein Jahr, der für die Patienten mit stärkerer intialer Stressbelastung signifikant war. Ein langfristiger Effekt auf den ADS-Wert war nicht festzustellen
- depressive Symptome waren zwar direkt nach der siebentägigen Trainingstherapie, nicht aber nach mehreren Monaten oder einem Jahr verbessert. Auch hier hatte die TRT aber einen signifikanten Langzeiteffekt bei denjenigen mit deutlicher depressiver Symptomatik zu Studienbeginn. Nur bei Frauen spielte die Dauer der Tinnnitusbeschwerden eine Rolle: Je länger die Beschwerden bereits bestanden, umso geringer war der Effekt auf die Beeinträchtigung durch Tinnitus.

Fazit: Mit einer intensiven siebentägigen TRT ließen sich langfristig allgemeine und krankheitsspezifische Belastungen wirksam vermindern, wobei Patienten, die initial stark unter dem Tinnitus litten, am stärksten profitierten.

fk

Seydel C et al. Long-Term Improvement in Tinnitus after Modified Tinnitus Retraining Therapy Enhanced by a Variety of Psychological Approaches.

Audiol Neurootol. 2009; 15: 69-80

\section{Kommentar}

In Anbetracht der praktisch fehlenden Wirksamkeit von medikamentösen und nur vereinzelt operativen Therapien beim chronischen Ohrgeräusch gilt das Therapieziel „Linderung des Leidens durch Defokussierung und Habituation" als die derzeit effektivste und weit verbreiteteste Behandlungsmethode. Nach einem Review des Cochrane-Instituts (2007) erfüllt die kognitive Verhaltenstherapie die Kriterien der evidenzbasierten Medizin (Stufe lb) mit den größten Effektstärken. Im ambulanten Sektor sind Counselling sowie kognitive Techniken, Aufmerksamkeitslenkung und Demystifizierung des Tinnitus Hauptbestandteil und wesentlicher Wirkfaktor der TRT.Um sich hier von den wenig anspruchsvollen Behandlungssettings der angloamerikanischen TRT abzugrenzen, hat 2000 eine Arbeitsgruppe der ADANO festgelegt, dass die TRT in Deutschland von einem interdisziplinären Behandlungsteam von HNOArzt und ausgebildeten Psychotherapeuten (Ärztlicher Psychotherapeut oder klinischer Psychologe) durchzuführen ist. Der Audiologe oder Hörgeräte-Akustiker unterstützt die Behandlung bei indizierten Fällen. Passive apparative Versorgung (Rauschgenerator, Hörgerät) gehört hier zu den adjuvanten Therapiebestandteilen.

Diese überzeugende Datenlage hat sich auch in der AWMF-Leitlinie Tinnitus (2010) niedergeschlagen: Für den chronischen Tinnitus mit niedrig- bis mittelgradiger Belastung wird eine Behandlung nach den Grundsätzen des TRT/ADANO als Mittel der ersten Wahl empfohlen!

Seydel et al. gelingt es mit einem kontrollierten Design, die Effektivität der TRT/ADANO in einer randomisierten prospektiven Studie zur belegen. Es wird damit unterstrichen, dass TRT/ADANO ein effektives und sinnvolles Behandlungskonzept darstellt, um mittelgradig Tinnitus-Belasteten zu helfen. In Deutschland gibt es circa 78 Therapiezentren, die nach einem ähnlichen Konzept Tinnitus-Betroffenen zur Verfügung stehen und nach Kenntnis des jährlich tagenden Qualitätszirkel der Deutschen Tinnitus-Liga (DTL) effektive Hilfe anbieten. Im Zweifelsfall kann die DTL, Sektion Qualitätszirkel TRT, Auskunft über seriöse Anbieter geben.

Es ist höchste Zeit, dass ein bewährtes Behandlungskonzept in die Regelversorgung der GKV eingebunden wird. Allerdings ist noch nicht absehbar, wie ein solches interdisziplinäres Therapiepaket sektorenübergreifend in das Erstattungssystem eingebunden werden kann. Viele Betroffene müssen daher selbst für ihre Gesundheit in die Tasche greifen. Dieses nach wie vor schwelende Ärgernis für Anbieter und Patienten kann aktuell nur über eine individuelle Kostenerstattung geregelt werden oder-wie vereinzelt realisiert - können die Kosten im Rahmen einer integrierten Versorgung (z.B. Bad Gandersheim, Bielefeld) abgefedert werden.

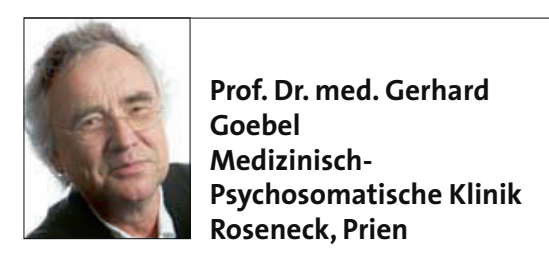

